\title{
Estimation de volume commercial du bois de Pterocarpus erinaceus Poir. (Fabaceae) dans les zones sahélo-soudaniennes et Soudaniennes du Niger et du Burkina Faso (Afrique de l'ouest)
}

\author{
Habou RABIOU1*, Babou André BATIONO33, Kossi Novigno SEGLA4, Abdoulaye DIOUF², Kossi \\ ADJONOU4, Adzo Dzifa KOKUTSE4, Raoufou RADJI4, Ali MAHAMANE (1,5), Kouami KOKOU4, Mahamane \\ SAADOU $(1,5)$ \\ 1: Université de Maradi, faculté des Sciences et techniques. BP 465 Maradi - NIGER \\ 2: Université de Maradi, faculté des Sciences du sol et de télédétection. BP 465 Maradi - NIGER \\ 3 : Institut de l'Environnement et de Recherches Agricole (INERA) du Burkina Faso 04 BP 8645 Ouagadougou 04 \\ 4 : Laboratoire de botanique et écologie végétale Université de Lomé Faculté des Sciences BP 1515, Lomé Togo. \\ 5: Laboratoire Garba Mounkaila, Département de Biologie, Université Abdou Moumouni, Faculté des Sciences et \\ Techniques. BP 10662 Niamey-Niger \\ Auteur correspondant : RABIOU Habou, mail : rabiouhabougarba@yahoo.fr
}

Original submitted in on $9^{\text {th }}$ February 2015. Published online at www.m.elewa.org on 31st March 2015 http://dx.doi.org/10.4314/jab.v87i1.13

\section{RÉSUMÉ}

Pterocarpus erinaceus est une espèce exploitée et menacée dans toute la zone Sahélo-soudanienne de l'Afrique de l'ouest. L'espèce est utilisée en sculpture, en menuiserie et surtout pour le bois exporté.

Objectif: L'objectif de cette étude est la construction de Tarif de cubage de Pterocarpus erinaceus et la quantification de volume de bois commercial dans 7 forêts de différentes zones agro-écologiques du Burkina Faso et du Niger.

Méthodologie et résultats : L'échantillon est composé de deux transects perpendiculaires en bande dans chaque forêt et tous les individus du Pterocarpus erinaceus ayant un diamètre supérieur à $5 \mathrm{~cm}$ ont été mesurés. Pour ce faire la hauteur du fût et le diamètre à $1,30 \mathrm{~m}$ du sol, à la base de l'arbre et la partie supérieure de l'arbre à la base de première ramification ont été mesurés afin de calculer le volume du fût et établir le tarif de cubage. Les résultats ont révélé que le meilleur modèle de tarif de cubage de Pterocarpus erinaceus est de type puissance. Les tiges de diamètre compris entre $20 \mathrm{~cm}$ et $50 \mathrm{~cm}$ sont les plus représentés dans la majorité des forêts et c'est dans cette classe que le volume de bois commercial est concentré.

Conclusion et application des résultats: Les forêts sahélo-soudaniennes et soudaniennes disposent d'important potentiel ligneux de Pterocarpus erinaceus bien que l'espèce est menacée par des pratiques diverses avec comme conséquence une faible régénération. 


\section{ABSTRACT}

Pterocarpus erinaceus is an exploited and threatened species in all the Sudano-Sahelian zone of West Africa. The species is used in sculpture, carpentry and especially for exported timber.

Objective: The objective of this study is the construction of volume tables for Pterocarpus erinaceus and quantifying the volume of commercial timber in 7 forests from different agro-ecological zones of Burkina Faso and Niger.

Methodology and results: The sample consists of two perpendicular belt transects in each forest and all individuals of Pterocarpus erinaceus with a diameter greater than $5 \mathrm{~cm}$ have been measured. Measurements were made on the height and diameter of the trunk at $1.30 \mathrm{~m}$ from the ground, at the bottom of the tree and the upper part of the tree to the base of first branch in order to calculate the volume of the trunk and draw the volume table. The study revealed that the best model of volume table for Pterocarpus erinaceus is power type. Stems with a diameter between $20 \mathrm{~cm}$ and $50 \mathrm{~cm}$ are most represented in the majority of forests and it is in this class that the volume of commercial timber is concentrated.

Conclusion and application of results: The Sudano-Sahelian and Sudanian forests have important timber potential of Pterocarpus erinaceus although the species is threatened by various practices with consequent poor regeneration

\section{INTRODUCTION}

La forêt africaine couvre 650 million d'hectares et représente plus de $17 \%$ de la forêt mondiale (FAO (1995). Ces écosystèmes génèrent des devises et services environnementaux très importants, contribuant ainsi au développement culturel et socioéconomique mondial. Selon FAO (1995), l'exploitation forestière reste un facteur de développement économique et contribue à $2,7 \%$ du PIB dans dix grands pays tropicaux producteurs.

La situation est cependant de plus en plus critique car l'exploitation se concentre sur un nombre restreint d'essences de bois d'œuvre de qualité souvent exploité de façon frauduleuse sans aucun respect des principes d'aménagement (Ganaba et al., 1998). Pterocarpus erinaceus Poir. (Fabaceae) appelé aussi Vène, est l'une de ces espèces (Adjonou et al., 2010). L'espèce est l'un des meilleurs bois d'artisanat (Touré, 2001), utilisée en sculpture, en menuiserie et surtout pour le bois exporté. En conséquence, $P$. erinaceus est actuellement menacée dans toutes les zones forestières et les parcs agroforestiers de la zone

\section{MATÉRIEL ET MÉTHODES}

Sites d'études : Au Burkina, quatre forêts ont fait l'objet d'investigation dans le cadre de cette étude.

$>\quad$ Zone Soudanienne (Burkina Faso): L'ensemble des (4) sites prospectés au Burkina Faso appartiennent à la zone soudanienne. II s'agit de la forêt sahélo-soudanienne et soudanienne (Nacoulma, 2012 ; Deniveau, 1999 ; Gaoué et Ticktin, 2008) pour cause d'exploitation incontrôlée, les feux de brousse et la baisse de la pluviométrie. L'aménagement des peuplements de Pterocarpus erinaceus encore disponible dans son aire naturelle nécessite la quantification de la ressource en vue de planifier et exécuter les actions visant l'indispensable conciliation entre l'utilisation et la conservation de la biodiversité. C'est dans cette optique que la présente étude s'est fixée comme objectif général de contribuer à l'élaboration des normes de gestion afin de doter les gestionnaires d'un outil pour un aménagement cohérent des peuplements de $P$. erinaceus dans les zones sahélo-soudaniennes et Soudaniennes du Burkina Faso et du Niger. Spécifiquement, il s'agit de construire le Tarif de cubage de Pterocarpus erinaceus et de quantifier le volume de bois commercial dans 7 forêts de différentes zones agro-écologiques du Burkina Faso (4 forêts) et du Niger (3 forêts).

de Tiogo, Laba, Cassou, et Saponé. Sur le plan phytogéographique, ces forêts sont situées dans la zone de transition entre les zones nord-soudanienne et sudsoudanienne (Fontès et Guinko, 1995). La forêt classée de Tiogo, de coordonnées géographique $12^{\circ} 13^{\prime} \mathrm{N}$ et 
$2^{\circ} 42^{\prime} \mathrm{W}$, est située sur l'axe Koudougou-Dédougou à 40 $\mathrm{km}$ de la ville de Koudougou. La forêt classée de Laba quant à elle, est située sur l'axe Ouagadougou-BoboDioulasso à $150 \mathrm{~km}$ de la ville de Ouagadougou, et a pour coordonnées géographiques $11^{\circ} 40^{\prime} \mathrm{N}$ et $2^{\circ} 50^{\prime} \mathrm{W}$. Les forêts de Saponé et de Cassou sont situées sur l'axe Ouagadougou-Léo-Frontière du Ghana, respectivement à $30 \mathrm{~km}$ et $130 \mathrm{~km}$ de Ouagadougou. La hauteur moyenne d'eau tombée entre 1992 et 2010 est de $827 \pm 169 \mathrm{~mm}$ à Tiogo et $907 \pm 157 \mathrm{~mm}$ à Laba (Sawadogo, 2009). La pluviométrie oscille entre 900 et $1200 \mathrm{~mm}$ par an à Cassou et est de $806 \mathrm{~mm}$ à Saponé sur une période de 30 ans. La forêt classée de Tiogo couvre une superficie de 30389 ha et celle de Laba 18501 ha (Sawadogo, 2009). La forêt de Cassou couvre une superficie de 29515 ha et celle de Saponé couvre une superficie de 100 ha (Figure 1).

$>\quad$ Zone Sahélo-soudanienne (Niger) : Au Niger, 3 sites ont fait l'objet d'investigation. II s'agit de la réserve de faune de Tamou, Parc W et la forêt classée de Gorou Bassounga situé dans le département de Gaya. La réserve de Tamou se situe entre $12^{\circ} 28^{\prime}$ et $12^{\circ} 50^{\prime}$ de latitude Nord et $2^{\circ} 06^{\prime}$ et $2^{\circ} 24^{\prime}$ de longitude Est. Elle couvre une superficie de 76000 ha, la pluviométrie moyenne annuelle est de $606 \mathrm{~mm}$ au poste pluviométrique de Tamou (1988 à 2007). La température moyenne annuelle est de l'ordre de $36,7^{\circ} \mathrm{C}$. La végétation de la réserve de faune de Tamou est une mosaïque de forêts claires, de savanes arborées, savanes arbustives et savanes herbeuses (Saadou, (1990) ; Couteron et al. (1992)). Le parc régional du W est une aire protégée transfrontalière à cheval entre le Bénin, le Burkina Faso et le Niger, L'étude a été menée dans la portion du Niger située entre $11^{\circ} 00^{\prime}$ et $12^{\circ} 35^{\prime}$ de latitude Nord et $2^{\circ} 00$ et $3^{\circ} 50^{\prime}$ de longitude Est. Le climat est de type tropical sec, caractéristique de la zone des steppes et savanes. La température moyenne annuelle est de $30^{\circ} \mathrm{C}$ sur une période de 20 ans et une pluviométrie annuelle moyenne de 704,7 mm (Diouf et al., 2010). La forte de Gorou Bassounga se situe à l' extrême sud du Niger dans le département de Gaya, c'est la zone la plus arrosée du Niger, la pluviométrie moyenne annuelle calculée sur une période de 58 ans est de $740 \pm 119,5 \mathrm{~mm}$ à la station de Gaya (Dan guimbo, 2010) (Figure 1). La végétation de cette forêt est dominée par des forêts sèches basses sur les plateaux, les forêts claires sur les terrasses argileuses méridionales et les toposéquences de vallées et les savanes dans les vallées sèches et sur les dunes fixées surplombant les vallées (Larwanou, 1992).

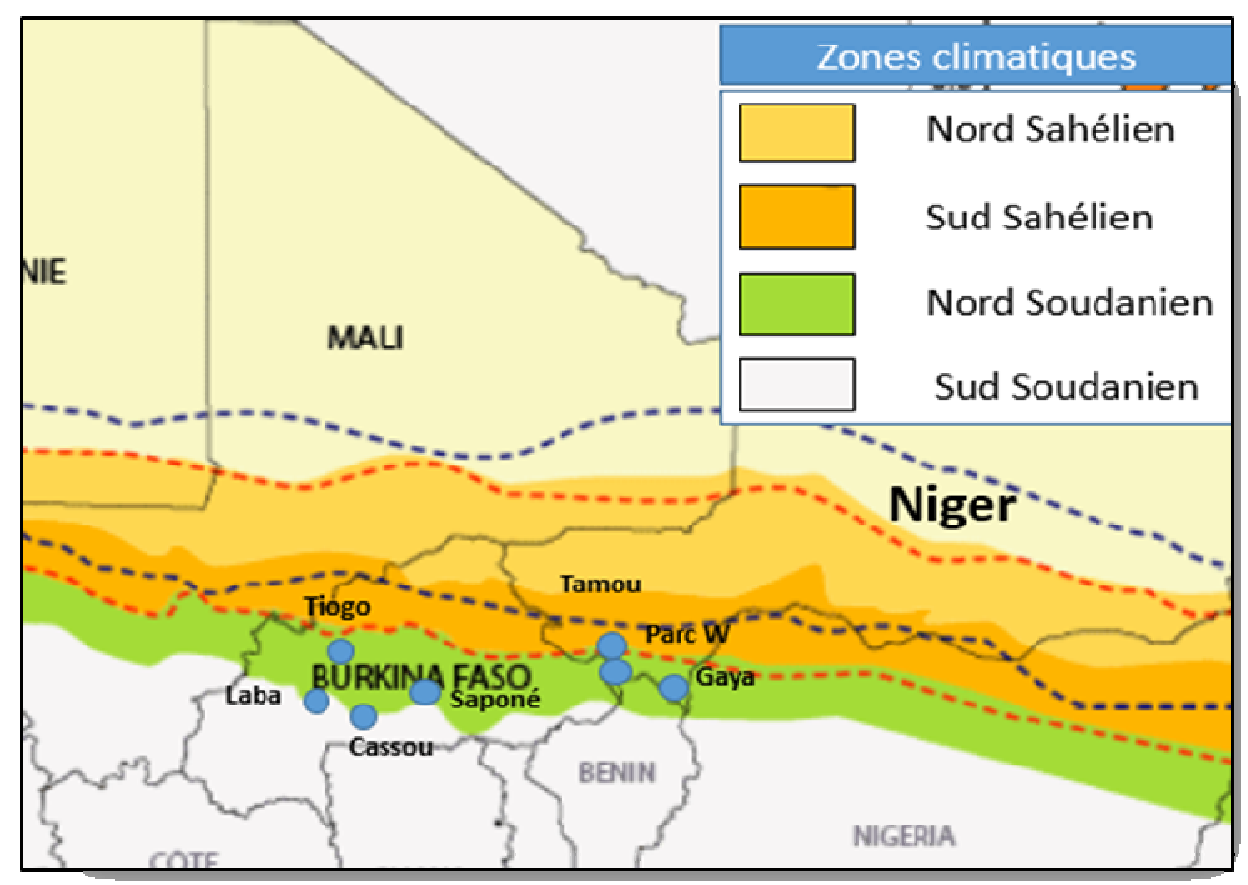

Figure 1: Carte de localisation des sites d'études (Source : Centre Régional Agrhymet (CRA), CSAO/ OCDE (2005)) 
Collecte des données : L'échantillonnage est axé sur des transects en bande d'environ $200 \mathrm{~m}$ de largeur et une longueur variables en fonction de la densité et de la superficie des sites couverts (Assogbadjo, 2005). La direction de l'azimut de marche a été fixée à l'aide d'un GPS, la distance entre l'arbre et la direction de l'azimut a été estimé à l'aide d'un télémètre laser pour éviter que l'arbre ne dépasse $100 \mathrm{~m}$ de part et d'autre de l'azimut. Les coordonnées de point de départ et point d'arrivée ont été relevées et la distance parcourue est estimée par le GPS. Dans cette bande tous les individus de Pterocarpus erinaceus de diamètre supérieur à $5 \mathrm{~cm}$ ont fait l'objet de mesures. Il s'agit des diamètres de tronc à $1,30 \mathrm{~m}$ du sol (D), à la base de pied au-dessus de contrefort (d1) et le diamètre à la base de la première branche (d2) et enfin la hauteur du fût (I), respectivement à l'aide d'un compas forestier et d'une perche graduée de $5 \mathrm{~m}$.

Traitement des données : Les deux diamètres (d1 et d2) et la hauteur de fût collectées sur les arbres de $P$. erinaceus ont permis de déterminer le volume de fût de chaque arbre. Le volume de fût (Vf) de l'arbre i est déterminé au moyen de la formule de Smalian comme suit :

$V_{f i}=\frac{\pi}{4} \operatorname{li}\left(\frac{d_{1 i}^{2}+d_{2 i}^{2}}{2}\right)$

$d_{1 i}$ et $d_{2 i}=$ diamètres des deux extrémités de l'arbre $i$, li = hauteur de la grume de l'arbre $i$. Cette formule permet d'estimer les volumes de bois des arbres sur pieds connaissant les deux diamètres extrêmes de la grume, contrairement à la méthode destructive (Fayole et al., 2013) ou par billon successif (Fonton et al., 2009).

Établissement des modèles : Les modèles de tarifs de cubage testés sont des tarifs à une entrée. Les raisons de ces choix résident dans le fait que dans toutes les formations naturelles investiguées hormis le Parc W, P.erinaceus fait l'objet d'une exploitation fourragère par émondage, la considération de la hauteur pourrait apporter des biais au niveau des modèles donc seuls le

\section{RÉSULTATS}

Caractéristiques dendrométriques des peuplements de $P$. erinaceus : Les résultats montrent que les sites des zones soudaniennes du Burkina Faso se distinguent par la densité élevée de $P$. erinaceus (Tableau 1). La forêt de Cassou présente une densité de 9,41 individus/ha, suivie de la forêt de Saponé avec une densité de 5,07 individus/ha. Les plus faibles densités sont observées dans les zones sahélo-soudaniennes du Niger avec des densités de 0,75 et 0,6 respectivement volume de fût et le diamètre ont été considérés. II s'agit des modèles linéaires et non linéaires suivants :

$$
\begin{array}{ll}
\text { - } & \text { Linéaire : } V f=a+b D \\
\text { - } & \text { Quadratique : } V f=a D^{2}+b D+c \\
\text { - } & \text { Cubique: } V f=a D^{3}+b D^{2}+c D+d \\
\text { - } & \text { Puissance : } V f=a D^{b} \\
& \text { Logarithmique : } V f=a+b \ln D
\end{array}
$$

L'analyse de la régression a été effectuée avec les données de volume de fût en fonction du diamètre à 1,30 $\mathrm{m}$ du sol. Le test de Durbin Watson a été utilisé pour vérifier l'autocorrélation des résidus studentisés choisi par validation croisée. La distribution de ces résidus autour de la droite de Henri a été analysée après un test de Ryan-joiner. Le test de conformité d'une moyenne a été utilisé pour vérifier si la moyenne résiduelle est nulle. Enfin, le test de Breush-Pagan nous a permis d'analyser l'hétérocédacité des résidus. Ainsi, le critère d'information d'Akaike (AIC) et le coefficient de détermination $\mathrm{R}^{2}$ ont été utilisés pour le choix de meilleur modèle. L'ensemble de ces tests ont été effectués pour les modèles puissances après transformations logarithmiques de Volume de fût et de diamètre afin de linéariser les modèles, le modèle ainsi obtenu est de type $\ln (\mathrm{Vf})=\ln (\mathrm{a})$ $+b \ln (D)$. Les données ont été analysées à l'aide de logiciel R 2.15 .3 et de son package R commander.

Estimation des volumes du bois à l'aide des modèles retenus : Les volumes du bois ont été estimés à l'aide des modèles retenus. Les centres des classes de diamètre ont servi d'entrée dans le modèle. Ainsi, pour chaque classe de diamètre, le volume du bois est calculé puis ramené à l'hectare. La somme des volumes de bois de toutes les classes observées donne le volume du bois total par hectare.

$V f c i=a D c i^{b}$

$\mathrm{Vf}$ (volume par ha) $=\sum \mathrm{Vfci}^{*} \mathrm{ni}$

avec

$\mathrm{ci}$ : centre de classe de diamètre $\mathrm{i}$; et $\mathrm{n}$ : la densité de classe i par ha.

pour Tamou et Gaya. Mais les peuplements ayant le plus gros diamètre moyen sont ceux du Parc W et de la forêt de Gaya au Niger avec des diamètres moyens respectifs de $25,49 \pm 7,8$ et $25,60 \pm 8,7 \mathrm{~cm}$. Ces différences sont statistiquement significatives $(P<0,001)$. Le peuplement du Parc $W$ se distingue également par la hauteur moyenne de fût $(4,35 \pm 1,50 \mathrm{~m})$ suivi de la forêt de Laba et de Tiogo en pleine Zone nord Soudanienne $(P<0,001)$. 
Rabiou et al. J. Appl. Biosci. Estimation de volume commercial du bois de Pterocarpus erinaceus Poir. (Fabaceae) dans les zones sahélo-soudaniennes et Soudaniennes du Niger et du Burkina Faso

Tableau 1 : Caractéristiques dendrométriques

\begin{tabular}{|c|c|c|c|c|c|}
\hline Pays & Sites & Densité/ha & Diamètre $(\mathrm{cm})$ & Hauteur fût (m) & Nombre \\
\hline \multirow{4}{*}{ Burkina Faso } & Cassou & 9,41 & $25,49 \pm 7,8 a$ & $3,53 \pm 1,05 a$ & 320 \\
\hline & Saponé & 5,07 & $25,60 \pm 8,7 a$ & $3,27 \pm 1,10 a$ & 272 \\
\hline & Tiogo & $2,26 a$ & $36,68 \pm 15,82 b$ & $3,68 \pm 1,67 b$ & 110 \\
\hline & Laba & $2,84 a$ & $33,63 \pm 10 b$ & $3,95 \pm 1,42 b$ & 181 \\
\hline \multirow{3}{*}{ Niger } & Parc W & 1,81 & $57,7 \pm 18,6$ & $4,35 \pm 1,50$ & 234 \\
\hline & Tamou & $0,75 b$ & $36,89 \pm 12,6 b$ & $3,65 \pm 0,90 b$ & 148 \\
\hline & Gaya & $0,6 b$ & $43,15 \pm 11,19$ & $3,24 \pm 1,14 a$ & 105 \\
\hline \multicolumn{2}{|c|}{ Probabilité } & $P<0,001^{* *}$ & $P<0,001^{* *}$ & $P<0,001^{* *}$ & $P<0,001^{* *}$ \\
\hline
\end{tabular}

Dans une même colonne seules les cases ayant les mêmes lettres n'ont pas de différences significatives et celles * : différence significative

Structure et densité des classes de diamètre: La structure des diamètres diffère d'un site à un autre (Figue 2). Les classes de diamètre de 70 à $100 \mathrm{~cm}$ ne sont observées qu'au niveau de Parc W. Les classes de diamètre comprises entre 20 à $30 \mathrm{~cm}$ sont prédominantes dans la forêt de Cassou et de Saponé. Toutes fois les classes de diamètre moyen d'environ $30 \mathrm{~cm}$ dominent toutes les forêts. Les individus jeunes sont remarquablement moins représentés dans les sites prospectés. Les structures en diamètres de tels peuplements sont caractéristiques des peuplements non stables. Le renouvellement n'est pas garanti.

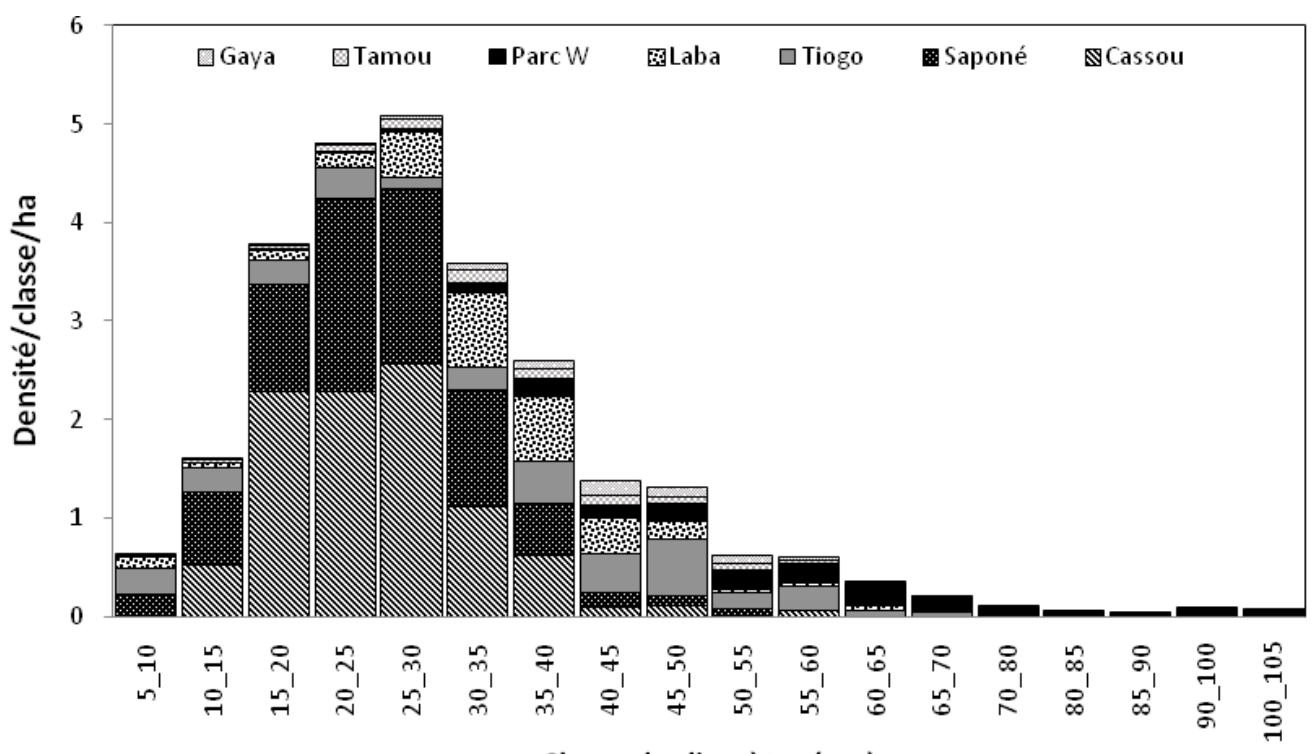

Figure 2 : Structure en diamètre des peuplements de $P$. erinaceus

Modèles allométriques de tarif de cubage : Le meilleur modèle de régression qui s'ajuste de façon significative est de type puissance (Tableau 2). En effet, la vérification de ces modèles est basée sur les conditions d'application ainsi que les différents tests de validité des modèles. Le coefficient de régression a et $b$ sont significatifs pour le modèle puissance testé et non significatifs pour les modèles quadratiques et cubiques bien que le coefficient de détermination $\mathrm{R}^{2}$ et l'AIC sont favorables à ces modèles ainsi que le residuel standard erreur RSE soit faibles. Le test de significativité de modèle montre que ces modèles sont globalement satisfaisants $(P<0,001)$. Les courbes ajustées aux histogrammes de distribution résiduelle sont de forme en cloche. Le test de Ryan- 
Joiner montre que les résidus suivent une distribution normale $(P>0,05)$ pour les deux modèles précités. L'examen de la distribution des résidus autour de la droite de Henri montre un ajustement linéaire. Ainsi la validité des modèles a été basée sur le critère d'akaike (AIC) et le coefficient de détermination $\mathrm{R}^{2}$. En effet, tous les modèles retenus sont des modèles qui présentent les critères d'informations d'Akaike (AIC) très faibles par rapport aux autres modèles (Tableau 2). Le coefficient de détermination $\mathrm{R}^{2}$ indique au niveau de toutes les forêts en zone sahélo-soudanienne qu' à plus de 71,6 à 92,4\% de la variation de volume de fût est due à la variation du diamètre à $1,30 \mathrm{~m}$. Les résultats montrent les relations qui existent entre la hauteur de fût et le diamètre à $1,3 \mathrm{~m}$ du sol. Les allures des courbes montrent clairement que les tendances ne sont pas linéaires (Figure 3).

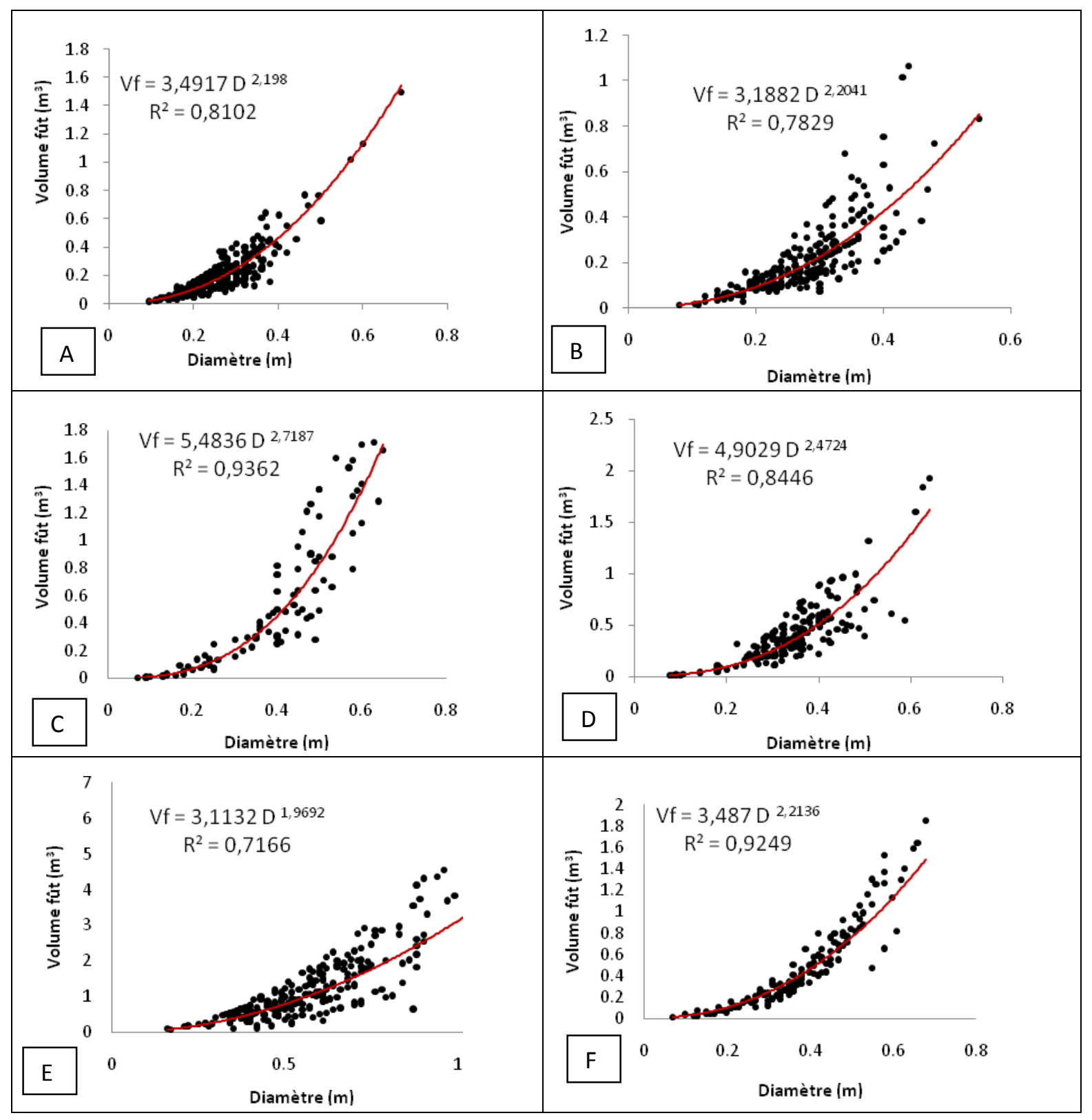




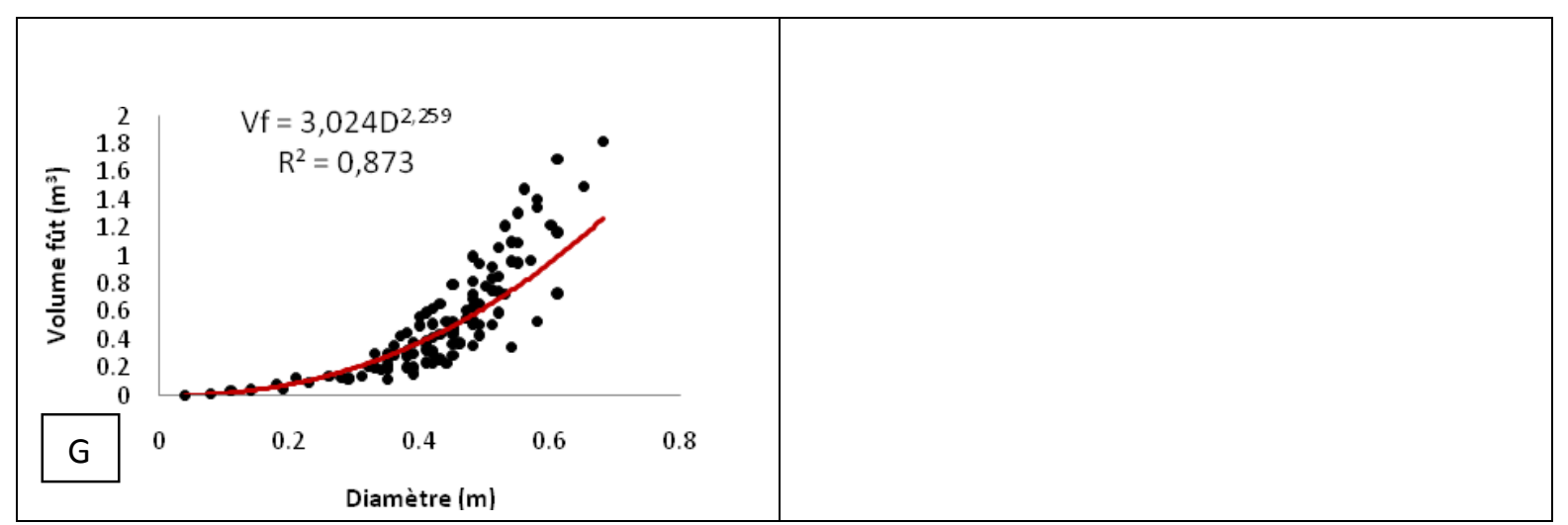

Figure 3 : Modèles de tarif de cubage A : Cassou ; B : Saponé ; C : Tiogo ; D : Laba ; E : Parc W ; F : Tamou ; G : Gaya.

Volume de bois commercial de $P$. erinaceus: Le volume de bois commercialisable par hectare est concentré au niveau des classes de diamètre de 20 à 35 $\mathrm{cm}$ au niveau de la forêt de Cassou et de la forêt de Saponé (Figure 4). La forêt de Tiogo se distingue par une concentration de volume de bois important au niveau des classes de diametre de 45 à $60 \mathrm{~cm}$. La forêt de Laba présente une concentration de bois de $P$. erinaceus dans les classes de diametre de $35-45 \mathrm{~cm}$. La reserve de faune de Tamou et la forêt classée de Gaya étant tres faible en densité de Pterocarpus erinaceus, ne présentent pas une grande variation. On constate cependant une concentration de bois au niveau des classes de diametre de 40 à $55 \mathrm{~cm}$. Le parc W héberge plus de volume de bois dans les plus grandes classes de diametre ( 60 à 80 $\mathrm{cm})$.

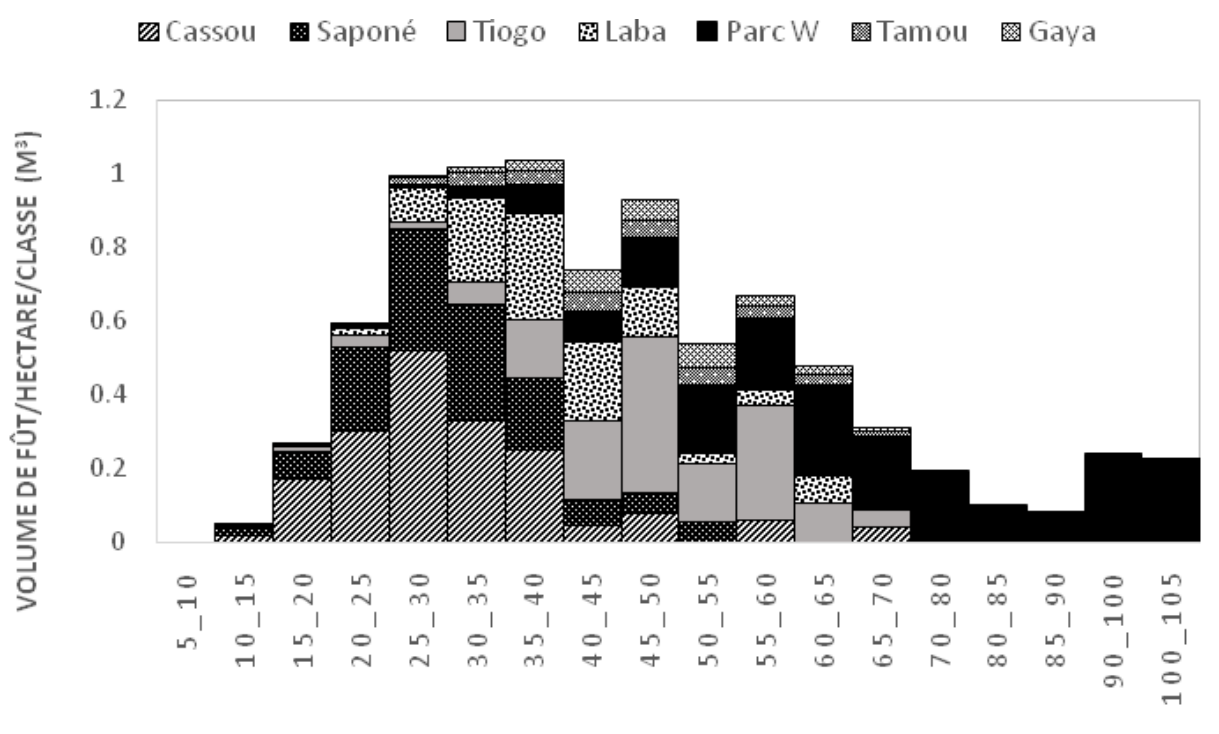

CLASSE DE DIAMETRE (CM)

Figure 4 : Volume de bois commercial de $P$. erinaceus par classe de diamètre 
Rabiou et al. J. Appl. Biosci. Estimation de volume commercial du bois de Pterocarpus erinaceus Poir. (Fabaceae) dans les zones sahélosoudaniennes et Soudaniennes du Niger et du Burkina Faso

Tableau 2 : Modèles allométriques de tarif de cubage

\begin{tabular}{|c|c|c|c|c|c|c|c|c|c|}
\hline \multirow{2}{*}{ Pays } & \multirow{2}{*}{ Sites } & \multirow{2}{*}{ Modèle } & \multicolumn{4}{|c|}{ Paramètres de régression (intervalle de confiance à $95 \%$ ) } & \multirow{2}{*}{$\mathbf{R}^{2}$} & \multirow{2}{*}{ AIC } & \multirow{2}{*}{ RSE } \\
\hline & & & a & b & c & d & & & \\
\hline \multicolumn{10}{|c|}{ Modèle $1(\mathrm{Vf}=\mathrm{aDb})$} \\
\hline \multirow{4}{*}{$\begin{array}{l}\text { Burkina } \\
\text { Faso }\end{array}$} & Cassou & $\mathrm{Vf}=3,491 \mathrm{D} 2,198$ & {$[2,950 ; 4,090]$} & {$[2,080 ; 2,315]$} & & & $R^{2}=0,810$ & $-357,6$ & 0,316 \\
\hline & Saponé & $\mathrm{Vf}=3,188 \mathrm{D} 2,204$ & {$[2,632 ; 3,857]$} & {$[2,065 ; 2,343]$} & & & $R^{2}=0,782$ & $-256,3$ & 0,345 \\
\hline & Tiogo & $V f=5,483 D 2,718$ & {$[4,604 ; 6,527]$} & {$[2,569 ; 2,868]$} & & & $R^{2}=0,936$ & $-76,1$ & 0,358 \\
\hline & Laba & $\mathrm{Vf}=4,902 \mathrm{D} 2,472$ & {$[4,083 ; 5,882]$} & {$[2,315 ; 2,628]$} & & & $R^{2}=0,844$ & $-159,1$ & 0,355 \\
\hline \multirow{3}{*}{ Niger } & Parc W & $\mathrm{Vf}=3,113 \mathrm{D} 1,969$ & {$[2,784 ; 3,476]$} & {$[1,808 ; 2,129]$} & & & $R^{2}=0,716$ & $-126,4$ & 0,421 \\
\hline & Tamou & $\mathrm{Vf}=3,487 \mathrm{D} 2,213$ & {$[3,098 ; 3,919]$} & {$[2,110 ; 2,317]$} & & & $R^{2}=0,924$ & $-230,7$ & 0,250 \\
\hline & Gaya & $V f=3,024 D^{2,259}$ & {$[2,557 ; 3,575]$} & {$[2,091 ; 2,427]$} & & & $R^{2}=0,873$ & $-91,6$ & 0,353 \\
\hline \multicolumn{10}{|c|}{ Modèle $2\left(V f=a D^{2}+b D+c\right)$} \\
\hline \multirow{4}{*}{$\begin{array}{l}\text { Burkina } \\
\text { Faso }\end{array}$} & Cassou & $V f=3,31 D^{2}-0,206 D+0,018$ & {$[2,772 ; 3,858]$} & {$[-0,550 ; 0,138]$} & $\begin{array}{c}{[-0,033 ;} \\
0,070] \\
\end{array}$ & & $R^{2}=0,847$ & $-850,07$ & 0,063 \\
\hline & Saponé & $V f=3,54 D^{2}-0,335 D+0,02$ & {$[2,265 ; 4,828]$} & {$[-1,071 ; 0,399]$} & $\begin{array}{c}{[-0,082 ;} \\
0,123]\end{array}$ & & $R^{2}=0,667$ & $-531,74$ & 0,090 \\
\hline & Tiogo & $V f=5,688 D^{2}-1,225 D+0,079$ & {$[3,658 ; 7,717]$} & {$[-2,714 ; 0,263]$} & $\begin{array}{c}{[-0,171 ;} \\
0,330]\end{array}$ & & $R^{2}=0,801$ & $-12,038$ & 0,220 \\
\hline & Laba & $V f=4,305 D^{2}-0,511+0,041$ & {$[2,853 ; 5,757]$} & {$[-1,535 ; 0,513]$} & $\begin{array}{c}{[-0,139 ;} \\
0,221] \\
\end{array}$ & & $R^{2}=0,689$ & $-143,31$ & 0,160 \\
\hline \multirow{3}{*}{ Niger } & Parc W & $V f=4,363 D^{2}-0,988 D+0,229$ & {$[2,919 ; 5,806]$} & {$[-2,828 ; 0,851]$} & $\begin{array}{c}-0,326 ; \\
0,785]\end{array}$ & & $R^{2}=0,725$ & 367,138 & 0,526 \\
\hline & Tamou & $V f=5,162 D^{2}-1,187 D+0,119$ & {$[4,250 ; 6,075]$} & $\begin{array}{c}-1,893 ;- \\
0,481]\end{array}$ & $\begin{array}{c}{[-0,009 ;} \\
0,249]\end{array}$ & & $R^{2}=0,909$ & $-215,74$ & 0,114 \\
\hline & Gaya & $V f=6,420 D^{2}-2,151 D+0,199$ & {$[4,794 ; 8,047]$} & $\begin{array}{c}{[-3,420 ;-} \\
0,882]\end{array}$ & $\begin{array}{r}{[-0,047 ;} \\
0,446] \\
\end{array}$ & & $\mathrm{R}^{2}=0,784$ & $-54,500$ & 0,182 \\
\hline \multicolumn{10}{|c|}{ Modèle $3\left(\mathrm{Vf}=a \mathrm{D}^{3}+b \mathrm{D}^{2}+\mathrm{cD}+\mathrm{d}\right)$} \\
\hline \multirow{3}{*}{$\begin{array}{l}\text { Burkina } \\
\text { Faso }\end{array}$} & Cassou & $V f=3,477 D^{3}-0,410 D^{2}+0,973-0,093$ & {$[0,204 ; 6,751]$} & {$[-3,957 ; 3,137]$} & $\begin{array}{c}{[-0,188 ;} \\
2,135]\end{array}$ & $\begin{array}{r}{[-0,210 ;} \\
0,024]\end{array}$ & $\mathrm{R}^{2}=0,848$ & $-852,47$ & 0,063 \\
\hline & Saponé & $V f=-3,835 D^{3}+6,977 D^{2}-1,285 D+0,1$ & {$[-13,48 ; 5,811]$} & {$[-1,745 ; 15,69]$} & $\begin{array}{r}{[-3,783} \\
1,213]\end{array}$ & $\begin{array}{r}{[-0,125 ;} \\
0,327]\end{array}$ & $R^{2}=0,666$ & $-530,36$ & 0,090 \\
\hline & Tiogo & $V f=-2,673 D^{3}+8,636 D^{2}-2,185 D+$ & {$[-15,89 ; 10,54]$} & {$[-6,079 ; 23,35]$} & {$[-7,161 ;$} & {$[-0,325$} & $R^{2}=0,799$ & $-10,20$ & 0,221 \\
\hline
\end{tabular}


Rabiou et al. J. Appl. Biosci. Estimation de volume commercial du bois de Pterocarpus erinaceus Poir. (Fabaceae) dans les zones sahélosoudaniennes et Soudaniennes du Niger et du Burkina Faso

\begin{tabular}{|c|c|c|c|c|c|c|c|c|c|}
\hline & & 0,165 & & & $2,790]$ & $0,655]$ & & & \\
\hline & Laba & $\begin{array}{l}V f=9,569 D^{3}-5,723 D^{2}+2,621 D- \\
0,237\end{array}$ & {$[0,506 ; 18,63]$} & {$[-15,33 ; 3,883]$} & $\begin{array}{c}{[-0,514} \\
5,756]\end{array}$ & $\begin{array}{c}{[-0,556 ;} \\
0,081]\end{array}$ & $R^{2}=0,695$ & $-145,7$ & 0,159 \\
\hline \multirow{3}{*}{ Niger } & Parc W & $\begin{array}{l}\mathrm{Vf}=-0,106 \mathrm{D}^{3}+4,566 \mathrm{D}^{2}-1,107 \mathrm{D}+ \\
0,25\end{array}$ & {$[-5,715 ; 5,502]$} & $\begin{array}{l}{[-6,247 ;} \\
15,380]\end{array}$ & $\begin{array}{c}{[-7,630 ;} \\
5,415]\end{array}$ & $\begin{array}{r}{[-0,850 ;} \\
1,486]\end{array}$ & $R^{2}=0,724$ & 369,13 & 0,527 \\
\hline & Tamou & $\begin{array}{l}\mathrm{Vf}=0,930 \mathrm{D}^{3}+4,110 \mathrm{D}^{2} \quad 0,829 \mathrm{D}+ \\
0,084\end{array}$ & {$[-4,679 ; 6,539]$} & {$[-2,302 ; 10,52]$} & $\begin{array}{c}{[-3,100 ;} \\
1,441]\end{array}$ & $\begin{array}{r}{[-0,165 ;} \\
0,333]\end{array}$ & $R^{2}=0,908$ & $-213,85$ & 0,114 \\
\hline & Gaya & $\begin{array}{l}V f=5,066 D^{3}+0,845 D^{2}-0,364 D+ \\
0,052\end{array}$ & {$[-4,706 ; 14,83]$} & {$[-10,03 ; 11,72]$} & $\begin{array}{c}{[-4,037} \\
3,308]\end{array}$ & $\begin{array}{c}{[-0,323 ;} \\
0,428]\end{array}$ & $\mathrm{R}^{2}=0,784$ & $-53,59$ & 0,182 \\
\hline
\end{tabular}

.Vf : volume du fût ; $\mathrm{D}$ : diamètre à $1,30 \mathrm{du}$ sol ; AIC : critères d'informations d'Akaike ; RSE : Erreur résiduelle standard ; $\mathrm{R}^{2}$ : coefficient de détermination 
Estimation du volume total de bois de $P$. erinaceus par forêt : Les modèles de tarifs de cubage élaborés ont été utilisés pour estimer les volumes des fûts par hectare puis extrapolés à l'échelle des forêts. La quantité de bois dépend essentiellement de la densité, de la superficie de site et également des caractéristiques dendrométriques du peuplement dans chacun des sites (Tableau 3). Les intervalles de prédiction des volumes du bois par ha au seuil de $95 \%$ sont consignés dans le tableau 3.

Tableau 3 : Estimation de la quantité de volume de bois de $P$. erinaceus

\begin{tabular}{llccc}
\hline Pays & Sites & $\begin{array}{c}\text { Superficie des sites } \\
\text { (ha) }\end{array}$ & $\begin{array}{c}\text { Volume de bois par } \\
\text { hectare }\left(\mathbf{m}^{3} / \mathbf{h a}\right)\end{array}$ & $\begin{array}{c}\text { Volume total de bois } \\
\text { commercial }\left(\mathbf{m}^{3}\right)\end{array}$ \\
\hline \multirow{4}{*}{ Burkina Faso } & Cassou & 29515 & 1,829 & 53975,3 \\
& Saponé & 100 & 1,361 & 136,1 \\
& Tiogo & 30389 & 1,545 & 46957,59 \\
& Laba & 18501 & 1,133 & 20966,23 \\
\hline \multirow{3}{*}{ Niger } & Parc W & 220000 & 2,028 & 446243,69 \\
& Tamou & 76000 & 0,324 & 24639,68 \\
& Gaya & 9970 & 0,29 & 2891,97 \\
\hline
\end{tabular}

\section{DISCUSSION}

Variabilité des paramètres structuraux des peuplements en fonction des zones agroécologiques: Les caractéristiques dendrométriques relatifs à la densité, le diamètre et la hauteur de fût des peuplements naturels de $P$. erinaceus ont fait l'objet de comparaison suivant les zones agro-écologiques. Les résultats montrent que la densité de $P$. erinaceus est plus importante dans les zones où les régimes pluviométriques sont plus favorables. En zones Sahéliennes, plus sèches, il ne subsiste que de vieux sujets de $P$. erinaceus. Ce qui explique la différence de diamètre de façon significative par rapport aux zones soudaniennes. Les diamètres observés dans cette étude confortent les résultats obtenus par Petit (2000) au Burkina Faso et Glélé et al. (2008) au Bénin. Par contre ces résultats ne concordent pas avec ceux de Bonkoungou (1999) selon lesquels, les arbres de P.erinaceus deviennent de plus en plus petits avec la latitude.

Distribution et densité des classes de diamètre : Les structures en diamètre de $P$. erinaceus dans les différentes forêts étudiées montrent que les individus de diamètres moyens d'environ 30 à $40 \mathrm{~cm}$ sont les plus représentés. Les individus jeunes sont presque absents même en conditions de protection intégrales. De même, les individus de très gros diamètres sont peu présents sur tous les sites sauf dans le Parc W où la majorité des individus ont un diamètre supérieur à $50 \mathrm{~cm}$. La structure en diamètres est en cloche sur tous les sites. Les mêmes allures en cloche pour la distribution des classes de diamètres ont été trouvées par Nacoulma (2012) dans le parc W du Burkina Faso, par Glélé et al. (2008) dans la forêt de Wari Maro au Bénin et par Adjonou et al. (2010) dans la réserve de faune d'Abdoulaye au Togo. L'allure de la distributions des classes de diamètre de $P$. erinaceus caractérisée par la quasi-absence de la population juvénile traduit une faible capacité de régénération (Bationo et al., 2005) indiquant ainsi que P.erinaceus est une espèce menacée même dans les aires où il est supposé être protégé (Dan Guimbo, 2010). Les volumes de bois commercial estimé par classe de diamètre et par hectare montrent que le volume de bois de P.erinaceus est concentré dans les tiges de diamètre moyen dans toutes les zones agro-écologiques. Cette configuration est le reflet de la structure en diamètres observés.

Modèles des Tarifs de cubage : Les tarifs de cubage des peuplements de $P$. erinaceus élaborés dans le cadre du présent travail sont de types puissances pour toutes les forêts et pour toutes les zones agro-écologiques du Niger et du Burkina Faso. L'observation minutieuse des intervalles de confiance des paramètres de régression a et $b$ montre des variations même au sein d'une zone agro-écologique. En effet, la forêt de Cassou et de Laba toutes appartenant à la zone sud Soudanienne montre l'impossibilité d'être regroupé du faite de la différence significative des intervalles de confiance de paramètres a et $b$. Ces différences peuvent s'expliquer par des conditions édaphiques différentes qui règnent dans ces deux forêts avec comme conséquence la différence significative entre le diamètre et la hauteur du fût (Dayamba, 2010). De plus la forêt de Cassou est 
constamment soumise au feu de brousse qui réduit considérablement le taux d'humidité du sol avec comme conséquence la frugalité des pieds de $P$. erinaceus, alors que la fréquence du feu est très peu observée au niveau de la forêt de Laba. Pour l'ensemble des modèles retenus, les coefficients de déterminations sont supérieurs à $70 \%$. L'examen des résidus a montré que ces modèles sont fiables. Le modèle de type puissance a été trouvé par Fayolle et al. (2013) sur trois espèces exploitées au Cameroun. Le tarif de cubage à une entrée dont l'équation est de type puissance a été utilisé en Afrique, notamment au Gabon sur Entandrophragma cylindricum et Erythrophleum suaveolens (Bilé, 1999; N'Gabou, 2011) et par le CTFT (1967) en Côte d'Ivoire. L'utilisation des modèles à deux entrées n'est pas pertinente pour une espèce comme $P$. erinaceus dont plus de $70 \%$ sont ébranchés voire complètement émondés chaque année pour nourrir les bétails. Dans ces conditions, les modèles intégrant la hauteur biaiseraient les résultats. Selon Fonton (2009) ces modèles sont pertinents pour estimer le volume individuel des arbres. Estimation de la quantité de volume de bois de $P$. erinaceus : Le volume de Pterocarpus erinaceus estimé dans les forêts a montré que ces formations végétales renferment une quantité non négligeable de bois dit de vène. La forêt de Cassou étant le site le plus arrosé montre un volume de bois d'environ $53975,30 \mathrm{~m}^{3}$. Cette

\section{CONCLUSION}

L'étude effectuée dans sept forêts suivant les zones agroécologiques de l'Afrique de l'ouest a montré que la densité de $P$. erinaceus est plus élevée dans les zones les plus arrosées au Burkina Faso. Le modèle de tarif de cubage le plus ajusté aux volumes de fût en fonction de diamètre à $1,30 \mathrm{~m}$ est de type puissance. $P$. erinaceus

\section{REMERCIEMENTS}

A la Commission de l'Union Économique et Monétaire de l'Afrique de l'Ouest (UEMOA) qui a financé cette recherche dans le cadre du programme "Appui à

\section{RÉFÉRENCES BIBLIOGRAPHIQUES}

Adjonou, K., Ali, N., Kokutse, A. D. et Kokou K., 2010. Étude de la dynamique des peuplements naturels de Pterocarpus erinaceus Poir. (Fabaceae) surexploités au Togo. Bois et Forêts des Tropiques, $n^{\circ} 306$ (1), pp 33-43.

Assogbadjo, A. E., Sinsin, B. \& Van Damme P., 2005b. Caractères morphologiques et production des capsules de baobab (Adansonia digitata $L$.) au Bénin. Fruits 60(5): 327-340. forte quantité s'explique essentiellement par la densité élevée de $P$. erinaceus $(9,4$ ind/ha). Le volume de bois de $P$. erinaceus dans la forêt de Tiogo est comparable à celui de la forêt de Cassou bien que sa densité soit le quart de celle de la forêt de Cassou. Cela s'explique par le fait que la forêt de Tiogo présente des gros individus de P.erinaceus de diamètre moyen de $36,68 \pm 15,82 \mathrm{~m}$ contre $25,49 \pm 7,8 \mathrm{~cm}$ à Cassou. La même situation explique le volume important observé dans le Parc W dont les individus observés présentent un diamètre moyen de $57,7 \pm 18,6 \mathrm{~cm}$. Le plus faible volume par hectare est observé dans la zone sahélo-soudanienne au Niger à Tamou et à Gaya. La raison de cette faiblesse s'explique essentiellement par la faible densité. Ces volumes par hectare sont de loin inférieurs à ceux trouvées par IRG (2004) dans la région de Tambacounda au Sénégal qui étaient de $8,85 \mathrm{~m}^{3} / \mathrm{ha}$ dans la forêt claire avec une densité de 47,7 ind/ha et de $2,92 \mathrm{~m}^{3} / \mathrm{ha}$ dans la savane arborée avec une densité de 22,6 ind/ha. Selon Fao (2005), le matériel sur pied par hectare d'une superficie forestière est un bon indicateur de la vitalité du matériel sur pied d'une forêt. Le volume mondial total du matériel sur pied est de $110 \mathrm{~m}^{3}$ par hectare (soit $11000 \mathrm{~m} 3$ par km²) et il n'a pas changé de manière significative au cours de ces 15 dernières années.

est caractérisée par une faible capacité de régénération dans les zones-écologiques étudiées lui conférant ainsi le statut d'espèce menacée dans ces zones. Les tiges de diamètre compris entre $20 \mathrm{~cm}$ et $50 \mathrm{~cm}$ sont les plus représentés dans la majorité des forêts et c'est dans cette classe que le volume de bois commercial est concentré.

I'Enseignement Supérieur (PAES)» dans Les pays membres.

Bationo B. A., Karim S. et Dourma M., 2005. Argumentaire pour l'étude et l'utilisation des marcottes et drageons dans les pays à faible couvert ligneux. AUF, sécheresse, volume $1 \mathrm{E}$, no3, $11 \mathrm{p}$.

Bilé Allogho J., 1999. Étude sur les ressources forestières du Gabon. Rome, Italie, Fao ; 4 WONG J. L. G., 1990. Forest resources 
management project temporary sample plot inventory computer program manual. ODA (UK).

Bonkougou E G., 1999. A quick guide to multipurpose trees from around the world. Arkansas, USA. (oneline), fact net. http://food-security.info/foodsecurity.info/Winrock\%20Archive/p_erinaceus.ht $\underline{\operatorname{Im}}$.

Couteron P., Kokou K. et Mahamane A., 1992.- Parc National du W (Niger) : typologie et cartographie de la végétation du Parc National et de la réserve de faune de Tamou, éd. MAB-UNESCO, $98 \mathrm{p}$.

CTFT, 1967. Étude de reboisement et de protection des sols - République de Côte d'Ivoire. Nogent-surMarne, France, Centre technique forestier tropical.

Dan Guimbo I., Mahamane A. et Ambouta K. J. M., 2010. Peuplement des parcs à Neocarya macrophylla (Sabine) Prance et à Vitellaria paradoxa (Gaertn. C.F.) dans le sud-ouest nigérien : diversité, structure et régénération. Int. J. Biol. Chem. Sci., 4 (5): 1706-1720.

Dayamba S. D., 2010. Fire, Plant-derived Smoke and Grazing Effects on Regeneration, Productivity and Diversity of the Sudanian Savannawoodland Ecosystem. Doctoral Thesis. Swedish University, $152 \mathrm{p}$.

Devineau J.L., 1999. Seasonal rhythms and phenological plasticity of savannah woody species in a follow farming system (southwest Burkina Faso), J. Trop. Ecol. 15 (1999) 497-513.

Diouf A., Nicola B., Mahamane A., Lejoly J., Saadou M., Bogaert J., 2010, Caractérisation de la structure spatiale des individus ligneux dans une brousse tachetée au sud-ouest du Niger. CNRS. Rev. Can. Rach. For. Vol. 40 : 827-835.

FAO, 1995. La sylviculture : élément déterminant d'un aménagement des forêts. Unasylva 181 (46): 23.

FAO, 2005. Global forest Global Forest Resources Assessment 2005, Progress towards sustainable forest Chapter 5: Productive functions of forest resources, 86p.

Fayolle A., Rondeux J., Doucet JL., Ernst G., Bouissou C., Quevauvillers S., Bourland N., Fétéké R., Lejeune P. (2013) : Réviser les tarifs de cubage pour mieux gérer les forêts du Cameroun. Bois et Forêts des Tropiques, 317 (3) : 35-49.

Fontès J., et Guinko S., 1995. Carte de la végétation et de l'occupation du sol du Burkina Faso.
Ministère de la Coopération Francaise: projet campus (88 313 101): $67 \mathrm{p}$.

Fonton N H., Yabi C C., Dah-Dovonon J Z., Adoko F K., Dotchamou T., 2009. Modélisation du volume du fût d'arbre pour une gestion durable des écosystèmes forestiers soudaniens. $\mathrm{N}^{\circ} 300(2)$ 95-100.

Ganaba S., Ouadba J.L., Bognounou O., 1998 Les ligneux à usage d'énergie en région sahélienne du Burkina Faso : Préférence des groupes ethniques, Sécheresse 9 (1998) 261-268.

Glèlè K. R. L., Sinsin B., Palm R., 2008. Etude dendrométrique de Pterocarpus erinaceus Poir. des formations naturelles de la zone soudanienne au Bénin. Agronomie africaine, 20 (3) : 245-255.

Goué OG., Ticktin T. 2008. Impact of bark and foliage harvest on Khaya senegalensis (Maliaceae) reproductive performance in Benin. J. Appl. Ecol. 45 :34-40.

IRG, 2004. Programme agriculture -gestion des ressources naturelles «Wula Nafaa . Rapport d'étude de la filière d'exploitation ; 27p.

Larwanou M., 1992 : Ecological survey of the GorouBassounga forest reserve, Gaya, Niger. B.Sc. Dissertation, Dept. Of forestry, University of Ibadan, 117p.

N'Gabou B. P., 2011. Origine et fiabilité des tarifs de cubage utilisés au Gabon pour les inventaires d'aménagement et d'exploitation. Rapport de stage. Libreville, Gabon, École nationale des eaux et des forêts.

Nacoulma B M I., 2012. Dynamique et stratégie de conservation de la végétation et de la phytodiversité du complexe écologique du Parc National du W du Burkina Faso. Université de Ouagadougou. 151p.

Petit S. 2000. Environnement, conduite des troupeaux et usage de l'arbre chez les agropasteurs peuls de l'ouest de Burkina Faso Approche comparative et systématique de trois situations: Barani, Kourouma, Ouangoldougou. Thèse de doctorat, Univ. D'Orléans. Farnce, 522p.

Saadou M. ; 1990. La végétation des milieux drainés nigériens à l'Est du fleuve Niger. Thèse de Docteur ès - Sciences Naturelles. - Université de Niamey. 395 p. + annexes.

Sawadogo, L. 2009. Influence de facteurs anthropiques sur la dynamique de la végétation des forêts classées de Laba et de Tiogo en zone 
Rabiou et al. J. Appl. Biosci. Estimation de volume commercial du bois de Pterocarpus erinaceus Poir. (Fabaceae) dans les zones sahélo-soudaniennes et Soudaniennes du Niger et du Burkina Faso

soudanienne du Burkina Faso. Thèse Doctorat d'Etat. Université de Ouagadougou: $142 \mathrm{p}$.

Touré $Y$., 2001. Étude des potentialités agro forestières de la multiplication et des usages de
Pterocarpus erinaceus Poir. en zone soudanienne du Burkina Faso ; mémoire IDR, $89 \mathrm{p}$. 